\title{
NEURAL MODELS OF HEAD-DIRECTION CELLS
}

\author{
PETER ZEIDMAN JOHN A. BULLINARIA \\ School of Computer Science, The University of Birmingham \\ Edgbaston, Birmingham. B15 2TT, UK
}

\begin{abstract}
After a review of the purpose and biological background of Head Direction Cells, and existing models of them, we introduce an improved neural model that integrates visual flow information with the vestibular inputs found in earlier models. Simulation results using real video inputs are presented to explore and validate the new model, the evolutionary advantages for the proposed arrangement are noted, its utility for use in robot controllers is discussed, and suggestions are made for further work in this area.
\end{abstract}

\section{Introduction}

The study of navigation is of interest in psychology, engineering, computer science and neurobiology. To navigate an environment successfully requires the integration of multi-modal sensory information, the maintenance of an accurate world model, and the ability to localize oneself and recover from mistakes. The neurological basis of such abilities has been studied extensively, and modelling the brain's methods for performing accurate navigation may also lead to the construction of an improved generation of mobile robots.

Successful navigation has three main functional requirements:

1. A Map: An internal representation of the environment needs to be generated, to allow sensory information to be associated with localities. This is the general function of Spatial Memory.

2. Knowledge of Current Location: O'Keefe \& Nadel (1978) described cells in rats' brains that act like a map of their environment. These Place Cells fire maximally when the rat is in a particular location. If moved to a different setting, the place cells spontaneously reconfigure, with each group of cells corresponding to a specific area of that environment (the "place field").

3. Heading Direction: In addition to one's map position, the heading direction is required for navigation. The neural representation of heading is via cells known as Head Direction (HD) Cells, and this "biological compass" is the focus of this paper. They were discovered by J.B. Ranck in 1984, and the first detailed study was published by Taube, Muller \& Ranck (1990).

The calculation of heading direction is a neural process that translates bodycentered sensory data (such as the retinal position of a visual cue) into a heading 


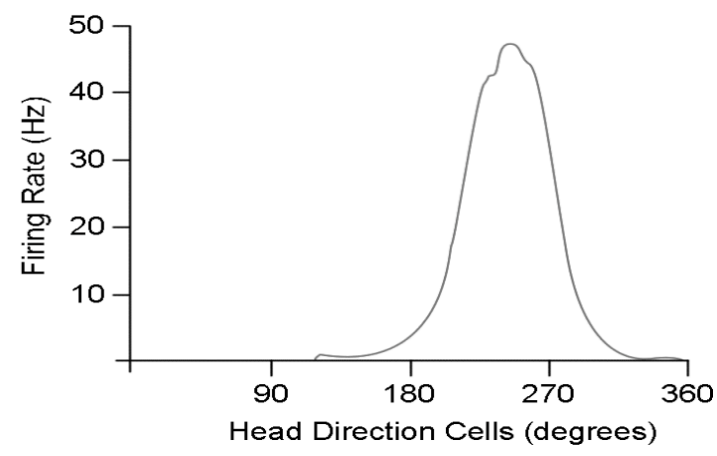

Figure 1: Typical Head Direction Cell firing profile. In this case the cell's preferred firing direction is 250 degrees (Sharp, Blair \& Cho, 2001).

in a world-centered model. The ability of the brain to produce these locationinvariant representations is an active research area and has implications both for our understanding of the brain and for the design of intelligent robots.

The following begins with an overview of the biological background behind Head Direction Cells, then introduces the sensory information used by the cells and the brain regions in which the cells are located. A review is given of the computational techniques and models that exist, and then our extended model is presented that integrates vestibular inputs with visual flow information.

\section{Biological Background}

\subsection{Head Direction Cells}

We have seen that navigation requires knowledge of heading, and that HD cells in the brain act as a biological compass. It is not a compass that uses magnetic fields. Instead, a combination of ideothetic (internally generated) and allothetic (externally generated) cues are used to update the heading representation.

Each Head Direction cell fires maximally for one heading direction, termed the preferred firing direction. Heading angles are world centered, i.e. measured from the perspective of a stationary external observer. Figure 1 shows a sample firing pattern from a single cell. Each cell's preferred firing direction is thought to be configured upon entering an environment, or given certain sensory stimuli. These directions are distributed equally around 360 degrees, and the cells operate as an integrated system: only one heading being represented at a time.

There are three major considerations for HD cell models:

- How should the HD representation be formed?

- How should the HD system track changes in its host's heading, so as to change which HD cells are firing at a given time? 
- What mechanisms can reconfigure the preferred firing directions of the HD cells, so that the cells come to represent a different heading?

We proceed by examining the different sensory inputs thought to contribute to HD cell firing, consider the differences in brain areas where HD cells are formed, and then move on to the regions of the brain where HD cells are located.

\subsection{Sensory Inputs}

By combining allothetic and ideothetic cues, the HD cells estimate the host's current heading. The primary sense employed by HD cells comes from the Vestibular System. Vestibular input is generated by structures in the inner ear, and signals the horizontal acceleration of the host's head in either direction. Experiments have shown that disabling the vestibular sense removes the head direction signal (Stackman, Clark \& Taube, 2002).

Vision may be divided into two categories: object (scene) recognition and optic flow. These two modalities of visual information are treated differently by the head direction system, and should therefore be considered independently.

Salient visual cues are used by the Head Direction system to set the current heading. This has been tested extensively via cue rotation experiments. Taube et al. (1990) placed a rat in a tall cylinder, with the only orientating cue being a piece of white card covering 90 degrees of the circumference. Rotating the cue by 90 degrees resulted in a near- 90 degree rotation in the preferred firing direction of every head direction cell, emphasizing not only that the cue was tracked, but also that the cells form a distributed representation of heading.

The HD cells must also track the gradual movement of the host over time, and this is where optic flow may be used. Results from Blair \& Sharp (1996) demonstrate the influence of visual flow on the HD system, and its interaction with the other senses. They constructed a cylinder to house the rat with a number of strips of white card positioned equidistantly around the cylinder's circumference as cues. The wall of the cylinder was rotated 90 degrees clockwise, with the floor stationary. This generated a conflict - visual stimuli made the rat believe it was turning counter-clockwise, while its vestibular system indicated it was stationary. Eight HD cells were recorded to discover how this sensory conflict would be resolved. Six out of eight of the cells showed no rotation in their preferred firing direction, meaning that only a quarter of cells were tricked by the moving wall. This demonstrated that, in a conflict situation, less credence is given to visual than vestibular input.

Empirical studies have shown that visual cues are used as a guide for orientation, but the lesser reliance on vision compared to vestibular sense leads to the hypothesis that optic flow is a gating mechanism for vestibular input. When in agreement, optic flow boosts the vestibular signal. When it is in 
contradiction, it weakens the vestibular signal but does not over-ride it.

\subsection{Brain Regions}

Head Direction cells were first discovered in an area of the brain known as the Post-Subiculum (PSc), but cells that fire as a function of the rat's heading have since been discovered in other brain areas. By understanding the purpose and interaction of these brain areas, a model of the system can be built. We now describe the relevant brain areas, with reference to suggested functions.

\subsubsection{Dorsal Tegmental Nucleus (DTN)}

Head Direction cells have not been found in the DTN, but it is considered to be a major input to the HD system, so we shall discuss it first. The majority of cells in the DTN have their activity correlated with the host's angular head velocity (Bassett \& Taube, 2001). Connections are received from horizontal vestibular canals; structures that signal head rotations. These signals are thought to be used for a number of purposes, including fixating eye position, as well as input for the head direction system. Asymmetric neurons consist of $27.3 \%$ of cells recorded by Bassett \& Taube (2001). These cells have a greater firing rate for either clockwise or counter-clockwise turns of the host's head. Less well understood are symmetric head-direction cells $(47.7 \%$ of those recorded), which fire equally given turns in either direction.

DTN is essential to the HD system: lesion studies show that damage to DTN causes loss of the HD signal in the ADN (Stackman \& Taube, 1998). It can be deduced that connections from DTN form the principal input to the HD system. Connections have been found projecting from DTN to area LMN, so LMN is probably the next area in the flow of information in the HD system.

\subsubsection{Lateral Mammillary Nucleus (LMN)}

The LMN has two major inputs: the DTN and the Post-Subiculum (PSc) which is thought to handle visual input to the system. HD cells in the LMN have preferred firing directions distributed evenly around 360 degrees. This input is vital to the HD system, and bilateral lesions cause loss of HD cell firing in area ATN, described below. HD cells in the LMN code for the host's head direction up to $40 \mathrm{~ms}$ in the future; further in the future than HD cells in the other brain areas described here. It is therefore reasonable to assume that LMN is near the source of the HD signal. Its function is thought to be part of an AttractorIntegrator network, along with DTN. This serves two purposes:

- Attractor: Maintains the population of HD cells in such a way that they represent only one heading direction. 
- Integrator: Integrates the movement of the host over time, so as to update the head direction signal accordingly.

LMN provides excitatory connections to the ATN, which is the next stage in the generation of the HD signal.

\subsubsection{Anterior Thalamus (ATN)}

The ATN contains the highest percentage of HD cells of the areas discovered. Bilateral lesions of LMN causes the ATN HD cells to lose their directional firing, implying that ATN sources its input from LMN. An interesting property of the ATN is that its firing is anticipatory - it represents the host's heading $20 \mathrm{~ms}$ in the future. Lesions to the ATN cause area PSc to lose its directional firing. Goodridge \& Taube (1997) suggested that "the AD projection may serve as a gating device that activates PoS [PSc] neurons sufficiently so that they are sensitive to the directional input from other structures". This hypothesis that ATN acts as a gating device is supported by the large percentage of HD cells it contains, and places the ATN at the centre of our model.

\subsubsection{Post-Subiculum (PSc)}

Lesion studies have shown that the PSc is not an essential component of the HD system, as it does not inhibit firing in the ATN. It is, however, required for visual cues to orientate the HD system (Goodridge \& Taube, 1997). It is reasonable to assume that visual input to the HD signal is either generated by the PSc, or as suggested by Goodridge, gated by the PSc. The PSc then sends connections back to LMN, completing the flow of information. However, it is not thought that the PSc sends visual information to LMN; instead it is believed that this reaches the ATN via a separate body: the retrosplenial cortex.

\section{Neural Modelling}

Artificial neural network models of the HD system operate as leaky integrators. By building up a representation over time, whilst not placing too great a reliance on any single sensory reading, the system is tolerant to noise and error.

\subsection{Continuous Attractors}

Attractor networks are a class of neural networks that are considered to be a good approximation to those in the HD system. Continuous Attractor Neural Networks (CANNs) consist of a set of nodes and weighted connections between them, and, depending on the implementation, there may be separate excitatory and inhibitory connections between nodes. The connection weights are preassigned to exhibit local cooperation and distal inhibition. This means that a 


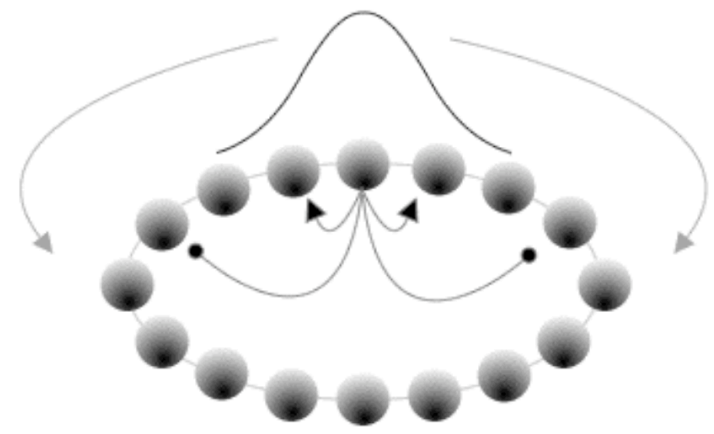

Figure 2: The Continuous Attractor Neural Network (CANN). Shaded circles represent neurons. A Gaussian "hill" of activity is shown over a number of neurons, representing their activation strength. Small pointed arrows represent local excitation; circular arrows represent distal inhibition.

node should excite its neighbours, increasing their activity level, but inhibit those further away so as to create a single peak of excitation (a "hill" of activity). The shape of this peak is determined by the weights, and its position is guided by the external inputs.

The key property of this network, that makes it useful for modelling biological systems such as Head Direction, is that the hill of activity persists after the input is removed. The network therefore acts as a short-term memory, integrating its input over time. The Gaussian hill can be shifted by supplying external input to neurons on either flank of the hill. Once input ceases, the hill comes to rest and is stable - the network is in an attractor state. The number of possible attractor states is determined by the number of neurons in the network.

Weights between nodes are a Gaussian function of their Euclidean distance:

$$
w_{i j}=\exp \left(-\left(\theta_{i}-\theta_{j}\right)^{2} / 2 \sigma^{2}\right)
$$

where $\theta_{i}$ is the preferred firing direction of neuron $i$, and $\sigma$ is the width of the excitatory or inhibitory effect. The firing properties of the CANN network are governed by three time $t$ dependent equations (Redish et al., 1996):

$$
\begin{gathered}
\tau \frac{d S_{i}(t)}{d t}=-S_{i}(t)+F_{i}(t) \\
F_{i}(t)=\frac{1}{2}\left(1+\tanh \left(V_{i}(t)\right)\right) \quad, \quad V_{i}(t)=\gamma_{i}+\sum_{j} w_{i j} S_{j}(t)
\end{gathered}
$$

where $S_{i}$ is the input received from neuron $i, F_{i}$ is the probability that neuron $i$ will fire, $V_{i}$ is the firing rate of neuron $i$, and $\gamma_{i}$ is a tonic inhibition term.

We next consider how such CANN networks have been used to implement HD models, and how we can use them to extend those past models. 


\subsection{Existing Models}

Redish et al. (1996) modelled the relationship between ATN and PSc. Each area was represented by a CANN, consisting of an excitatory and an inhibitory layer of nodes to maintain a single hill of activation. They based this on observations that PSc represents current heading, whereas ATN represents future heading. The model replicates this by using the ATN output to guide the PSc network.

Degris et al. (2004) designed an HD representation for a mobile robot based on the interaction between areas LMN and DTN in the rat brain. It used two sensory modalities: vestibular input to the DTN attractor network, and visual signals to the LMN for correcting drift. The dynamics of their system differed from that of most other authors, in line with the observation that no recurrent connections have been discovered in the LMN. They did not use excitatory connections between LMN units, instead establishing excitatory connections to the DTN network. This in turn inhibited LMN activity according to a Gaussian profile, such that a single hill of activity was maintained.

Once an attractor network has been set up with a hill of activity, the hill needs to be translated to reflect a shift in head direction. Skaggs et al. (1995) proposed two additional sets of neurons, called rotation cells, to guide the hill of activity around the network. Each rotation cell receives input from both its corresponding HD cell and the vestibular system. If the input is above a set threshold, the rotation cell will fire, transmitting its activation to the HD cells. A right-rotation cell transmits excitatory signals to the HD cells on the ring to its right; conversely left-rotation cells transmit only to HD cells to their left. The effect is to excite the HD cells on the appropriate flank of the hill of activity, given the vestibular input, and shift the hill of activity.

Skaggs et al. (1995) also provided the important concept of concentric rings of neurons, that integrate heading over time using multiple sensory modalities, but it was not described formally, nor verified experimentally, so further work was needed to test it. A more detailed model was proposed by Redish et al. (1996) with dual attractor networks, one representing the ATN and one the PSc. These networks were coupled by two types of connection: matching and offset. When activated by vestibular input, offset connections shifted the hill of activity, and thus the preferred firing direction of ATN cells. When vestibular input ceased, matching connections kept both networks aligned and stable.

This model generates tuning curves similar to the biologically plausible PSc and ATN cells, and the predictive relationship of ATN to PSc is faithfully replicated. Only vestibular input is considered; vision, self-motion and other senses are not mentioned. The problem with their model is that no recurrent excitatory connections have been found in the ATN, and PSc lesions do not disrupt ATN firing, so there remain doubts about the biological plausibility. 


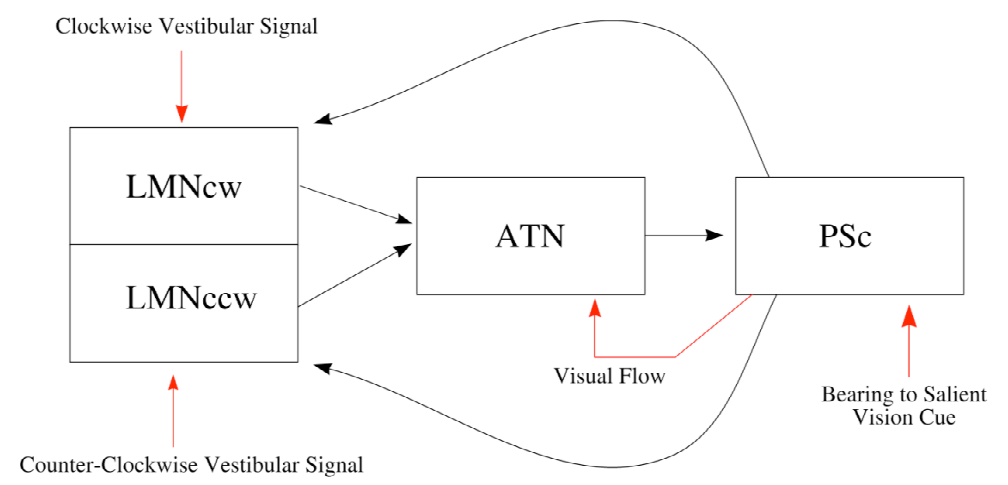

Figure 3: An overview of the extended HD model and the sensory inputs that control it. The introduction of sensory information is indicated by red/gray arrows, while the black arrows show connections between the attractor networks.

A different approach to shifting the CANN's preferred heading was used by Goodridge \& Touretzky (1994). Their model was based on observations that the tuning curves of ATN HD cells are distorted as a function of the host's angular velocity. They considered the interaction between three brain areas: LMN, ATN and PSc. LMN was represented by two attractor networks, one modulated by clockwise turns, the other by counter-clockwise turns. The ATN model combined the output of the two LMN networks into a single tuning curve which was then passed to the PSc. This tuning curve adjusted the firing direction of the PSc, which was taken to be the output of the system.

\subsection{The Extended Model}

The HD model of Goodridge \& Touretzky (1994) forms the basis for our model, but we need to introduce two major extensions:

- Visual flow information is transmitted from area PSc to ATN. This is in keeping with hypotheses of PSc's function in the HD system. The original Goodridge \& Touretzky (1994) model only accounted for vestibular input.

- The model of PSc is further extended to allow salient visual cues to orientate the HD system. The model is therefore also made practical for use in real-world robotics applications through the combination of three sensory modalities: visual flow, object recognition and vestibular input.

Figure 3 provides an overview of the model. PSC, LMNcw and LMNccw are CANNs. Each consists a layer of excitatory neurons and a single inhibitory neuron. Weights between excitatory neurons are initialized according to a Gaussian function of the distance between them. At each epoch or time-slice, the networks are updated in turn using discrete versions of the equations given in 
Section 3.1, as discussed in Weiner \& Taube (2005).

\subsection{Simulation Details}

Simulation software was developed to allow the model to be "placed" into a virtual cylindrical enclosure similar that used in the rat experiments of Taube et al. (1990), and to compare the results. This could later be extended to other types of behaviour testing enclosures, such as the Morris Water Maze.

Visual flow is provided to the model by a video of the enclosure. The model "sees" a 90 degree rotation clockwise, 180 degrees conter-clockwise, then 90 degrees to return to the starting position. The simulator converts this to a realvalued input parameter in the range [-1,1], with the sign indicating clockwise (positive) or counter-clockwise (negative) rotation. The visual flow algorithm is based on software by David Stavens (Stanford Artificial Intelligence Lab). A vector is generated for each salient feature in the image, where the bearing indicates direction of movement and length indicates the distance of that movement. Features are tracked from frame to frame and the velocity calculated.

Prior to running the model, simulated vestibular information needs to be associated with the visual stimuli. The input video is played and a numeric value manually associated with each frame. The video is then re-set and both visual and vestibular information are supplied to the model in real-time.

\subsection{Simulation Results}

\subsubsection{Tracking Vestibular Angular Velocity}

In the first experiment, simulated vestibular input complemented the visual stimulus provided to the model. The system was allowed 200 epochs to initialize before the stimuli were provided. The activity of the PSc network was recorded at each epoch, and compared against the actual heading of the agent.

Figure 4 shows the model and target outputs of the CANN model at two stages of the execution: after an 85 degree clockwise rotation, and then after a subsequent 180 degree turn counter-clockwise. The network's output underestimated the genuine rotation by about 6 degrees. This could be improved by varying the networks' connection strengths, or by using visual object recognition to periodically update the correct heading.

The velocity of a head turn in the model should reflect the angular velocity indicated by the sensory inputs. Changes in the CANN representation lagged behind the vestibular input by an average of 70 epochs.

\subsubsection{Optic Flow Integration}

A comparison was then made of the performance under sensory agreement and disparity. In the first scenario, visual flow supports the vestibular input. In the 


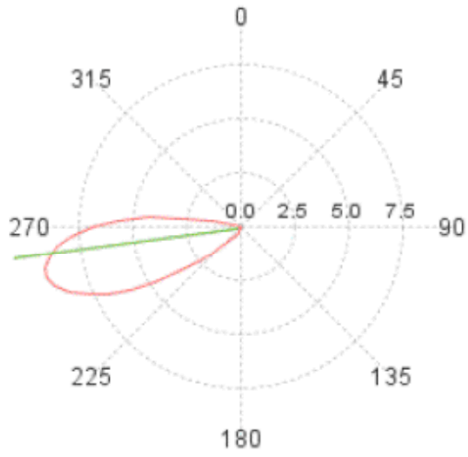

b.

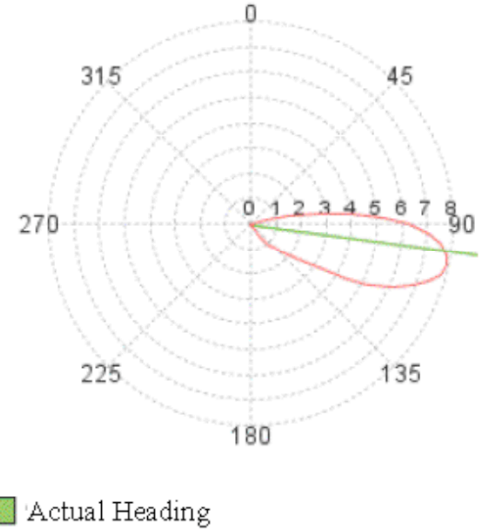

Figure 4: Directional heading graphs generated by the extended model. Chart a. shows the heading after an 85 degree $\mathrm{cw}$ turn, chart $\mathrm{b}$. after a 180 degree ccw turn.

second, the visual flow signal was inverted to establish a conflict situation.

Figure 5 shows the activity of the model given matching input data for: 90 degrees clockwise, 180 degrees counter-clockwise, 90 degrees clockwise. The comparative influence of the two sensory modalities can be seen more clearly when they are made to contradict. It can be seen in Figure 6, where the direction of travel indicated by optic flow has been purposefully inverted, that vestibular sense is treated with highest priority in a conflict situation. The conflicting optic flow signal reduces the effect of vestibular input, reducing the angular velocity of the model. The amount by which this reduction occurs is dependent on the parameters of the model, and may be adjusted accordingly.

\section{Conclusions}

The extended Head Direction model we have presented supports the hypothesis that visual flow acts as a gating function on vestibular inputs. The evolutionary advantage of such an arrangement is clear: visual information is far more subject to noise and misinterpretation than internally generated senses, and thus should be trusted less when disagreement occurs. The model tracks heading successfully and is a reasonable abstraction of real brain function.

The original motivation for this work was as inspiration for building robotic controllers, and the model offers two significant advantages in this application:

- The model degrades gracefully in the event of sensor damage, meaning that it will operate reasonably even if all external sensors are disabled.

- Additional senses with different modalities may be added, to increase resilience in the event of any individual sense becoming confused. 


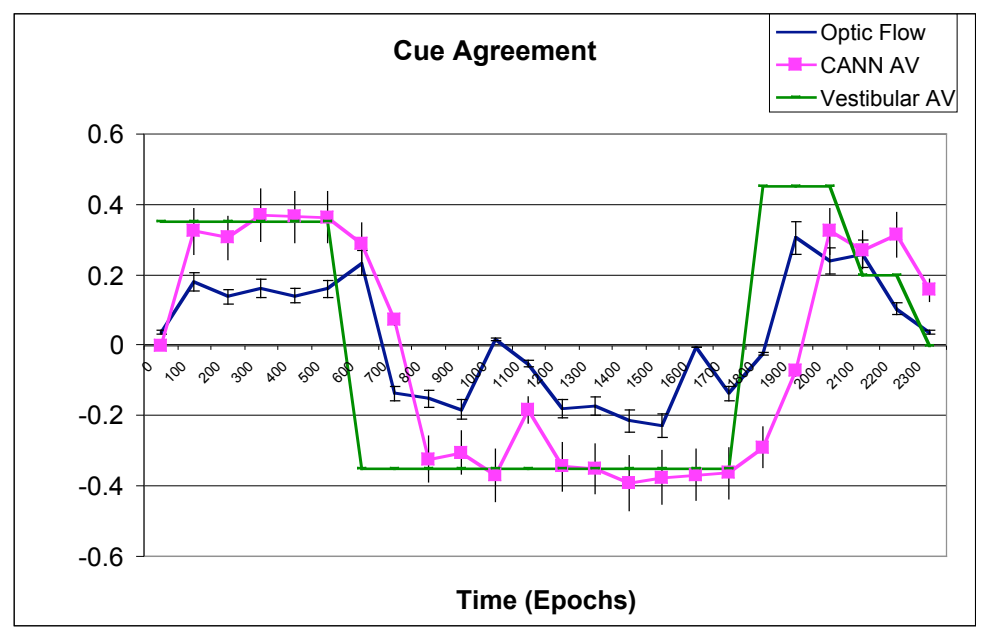

Figure 5: The angular velocity of the CANN in the presence of matching optic flow and vestibular input. (The angular velocity is scaled to lie in the range -1 to +1 .)

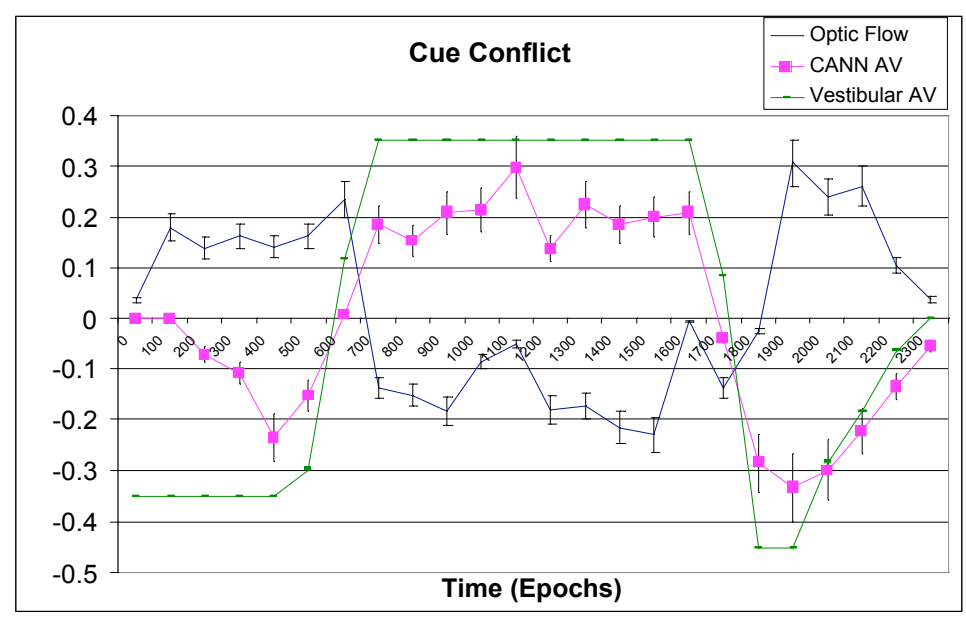

Figure 6: The angular velocity of the CANN network in a cue conflict situation.

There are two aspects of the current work that could be improved upon fairly easily. First, the optic flow input used in the simulation was very noisy, largely due to a poorly illuminated input video. Further testing should be conducted under improved conditions. Second, the parameters that control the attractor dynamics could be altered so as to avoid the system underestimating its current heading. Simulated evolution by natural selection is one approach that might prove helpful here, in which populations of attractor networks with different 
parameters repeatedly compete and reproduce leading to improved performance.

Future work will also need to consider further the precedence of the different senses during multi-modal integration, and quantify the point at which sensory disagreement leads to confusion about orientation. In the case of implementing Head Direction models for mobile robots, the task will be to automatically set appropriate thresholds for trusting or distrusting digital sensors.

\section{References}

Bassett, J.P. \& Taube, J.S. (2001). Neural correlates for angular head velocity in the rat dorsal tegmental nucleus. Journal of Neuroscience, 21, 5740-51.

Blair, H.T. \& Sharp, P.E. (1996). Visual and vestibular influences on head direction cells in the anterior thalamus of the rat. Behavioural Neuroscience, 110, 643-660.

Degris, T., Lacheze, L., Boucheny, C. \& Arleo, A. (2004). A spiking neuron model of head-direction cells for robot orientation. In Proceedings of the Eighth International Conference on the Simulation of Adaptive Behavior, from Animals to Animats, 255-263.

Goodridge, J.P. \& Taube, J.S. (1997). Interaction between the postsubiculum and anterior thalamus in the generation of head direction cell activity. Journal of Neuroscience, 17, 9315-9330.

Goodridge, J. \& Touretzky, D. (1994). Modeling attractor deformation in the rodent head-direction system. Journal of Neurophysiology, 83, 3402-3410.

O'Keefe, J. \& Nadel, L. (1978). The Hippocampus as a Cognitive Map. Oxford: Oxford University Press.

Redish, A.D., Elga, A.N. \& Touretzky. D.S. (1996). A coupled attractor model of the rodent head direction system. Network: Computation in Neural Systems, 7, 671-685.

Skaggs, W.E., Knierim, J.J., Kudrimoti, H.S. \& McNaughton, B.L. (1995). A model of the neural basis of the rat's sense of direction. Advances in Neural Information Processing Systems, 7, 173-180.

Stackman, R.W. \& Taube, J.S. (1998). Firing properties of head direction cells in rat anterior thalamic nucleus: Dependence on vestibular input. Journal of Neuroscience, 17, 9020-9037.

Stackman, R.W., Clark, A.S. \& Taube, J.S. (2002). Hippocampal spatial representations require vestibular input. Hippocampus, 12, 291-303.

Taube, J.S., Muller, R.U. \& Ranck, J.B. (1990). Head direction cells recorded from the postsubiculum in freely moving rats. II. Effects of environmental manipulations. Journal of Neuroscience, 10, 436-447.

Wiener, S.I. \& Taube, J.S. (Eds). (2005). Head direction cells and the neural mechanisms of spatial orientation. Cambridge, MA: MIT Press. 